\title{
Electrophysiological Studies of the Biceps Brachii Activities in Supination and Flexion of the Elbow Joint
}

\author{
Akira Naito, Michihiro Yajima*, Hidehiko \\ Fukamachi*, Koji Ushikoshi $\dagger$, Yasunobu Handa $\ddagger$, \\ Nozomu HoshimiYa§ and Yoshifusa Shimizu \\ Department of Anatomy, Shinshu University School of \\ Medicine, Matsumoto 390, *Kosei-Ren Kakeyu-Misayama \\ Hospital, Maruko-Machi 386-03, †Aizawa Hospital, \\ Matsumoto 390, $\ddagger$ Department of Anatomy, Tohoku \\ University School of Medicine, Sendai 980-77, and \\ $\S$ Department of Electronic Engneering, Faculty of \\ Engneering, Tohoku University, Sendai 980-77
}

Naito, A., Yajima, M., Fukamachi, H., Ushikoshi, K., Handa, Y., Hoshimiya, N. and Shimizu, Y. Electrophysiological Studies of the Biceps Brachii Activities in Supination and Flexion of the Elbow Joint. Tohoku J. Exp. Med., , 1994, 173 (2), 259-267 — Activities of the biceps brachii (long head: BiL, short head: BiS) on the elbow joint were studied using an electrical neuromuscular stimulation (ENS) and an electromyography (EMG). In ENS study, ENS to BiL or BiS was performed in seven volunteers. Before ENS, the volunteer relaxed the upper extremity on a table with the elbow extended and the forearm pronated. Then ENS-induced movements of the upper extremity were examined. Movements of elbow flexion and forearm supination were induced simultaneously by ENS to BiS in all volunteer and by ENS to BiL in six volunteers. ENS to BiL of one volunteer resulted in only elbow flexion. In EMG study, averaged EMGs of BiL and BiS during a to-and-fro motion from prone to supine of the forearm with holding a load were analyzed in eight volunteers. The volunteer acted the movements with keeping the elbow joint in different angles. Although an increase and a decrease of EMG activities in BiL and BiS were observed accompanied by the degree of forearm supination, patterns of changes in quantities of EMG activities to changing elbow angles varied from individual to individual. These findings seem to indicate that each human subject has an individual use of the biceps brachii for supination movements, while the action of the muscle on the elbow joint is similar among the subjects. —— long (BiL) and short (BiS) heads of the biceps brachii; electrical neuromuscular stimulation (ENS); electromyography (EMG); forearm supination; elbow flexion

Recent advance of the functional electrical stimulation (FES) has provided restoration of motor functions in paralyzed extremities caused by injury and disease of the upper motor neurons, i.e. spinal cord injuries or cerebrovascular

Received February 21, 1994; revision accepted for publication May 5, 1994. 
accidents (Liberson et al. 1961; Marsolais and Kobetic 1983; Nathan 1984). We have developed a computer-controlled multi-channel FES system using a percutaneous intramuscular electrode for selective activations of individual muscles (Handa and Hoshimiya 1987; Handa et al. 1987, 1989; Naito et al. 1988; Hoshimiya et al. 1989). The apparatus provided us well-coordinated control of movements including hand opening and grasping, wrist flexion and extension, and elbow flexion and extension of paralyzed upper extremities in quadriplegic or hemiplegic patients (Ichie et al. 1986; Matsushita et al. 1986; Fukamachi et al. 1987; Naito et al. 1987, 1990).

Movements of forearm pronation and supination are necessary to determine directions of the hand to objects (Kapandji 1986). The pronator teres and pronator quadratus, and the supinator and biceps brachii are main actiators for pronation and supination, respectively. We have been trying to control these movements in paralyzed upper extremities using FES to the pronator quadratus and the supinator. We confirmed that FES to the former provided smooth movements of forearm pronation. However, FES to the latter always induced simultaneous activations of the posterior interosseus nerve, which tunnels through the muscle, and resulted in inductions of wrist extension, finger extension, and thumb extension and abduction in addition to forearm supination. In this study, therefore, in order to use FES to the biceps brachii for controlling supination of paralyzed upper extremities, activities of the muscle were studied in normal volunteers using a electrical neuromuscular stimulation (ENS) and a electromyography (EMG).

\section{Materials ANd Methods}

\section{ENS study}

ENS-induced movements of the upper extremity were examined in seven normal volunteers ( 2 male and 5 female, 22-44 years of ages). Monopolar electrodes made of teflon-coated stainless steel wires (type 316, 75 micron in diameter) were implanted percutaneously into motor points of the biceps brachii (BiL: long head, BiS; short head) with 27 gauge injection-needles. Prior to electrode implantations, locations of the motor points were examined by surface electrical stimulation. Contractions of BiL and BiS induced by ENS were confirmed by palpating the original tendons of them. During the experiments, a volunteer was sitting on a chair and keeping the upper arm on a table with the shoulder flexed to about 90 degrees. Before ENS, he or she relaxed the upper extremity with the elbow extended and the forearm pronated. Then ENS-induced movements of the upper extremity were examined. ENS to each of BiL and BiS was performed more than 2 times in every volunteer. For ENS, we used amplitude modulated rectangular pulses $(-20-0 \mathrm{~V})$ with width of $0.2 \mathrm{msec}$, of which frequency was 20 $\mathrm{Hz}$ (Handa and Hoshimiya 1987; Handa et al. 1987; Hoshimiya et al. 1989). 


\section{$E M G$ study}

EMGs of BiL and BiS were recorded from eight volunteers (2 male and 6 female, 22-31 years of ages). Bipolar electrodes made of the same wires used for ENS study were implanted percutaneously into each muscle berry of BiL and BiS with 25 gauge injection-needles. Low noise amplifiers with high input impedance were used and EMGs were fed into a data recorder (RCD-928; Shinko, Tokyo) and a pen recorder (RECTI-HORIZ-8K; NEC San-ei, Tokyo), and then, rectified and averaged for analysis (Handa et al. 1987; Naito et al.1991).

A movement tested was a to-and-fro motion from prone to supine of the forearm (PS-movement). Volunteers performed the movement at different angles of the elbow joint (maximum extension, 30,60,90 and 120 degrees of flexion, maximum flexion). Each motion of supination and pronation was started at an experimenter's call and finished in several seconds. When supination was ended, he kept the forearm supine for more than 5 sec before pronation was started. Thus 18-25 sec were taken for each PS-movement. He also kept the forearm prone for several seconds before and after the movement. The movement was performed 2 times or more in each experimental situation. During experiments, he was sitting on a chair and keeping the upper arm on the table as well as in ENS analysis. In order to obtain enough EMG signals, a cylindrical object weighing $1.2 \mathrm{~kg}$ was held in the hand (Beevor 1903; Basmajian and Latif 1957; Basmajian and Deluca 1985). A potentiometer was used for indicating which motion was
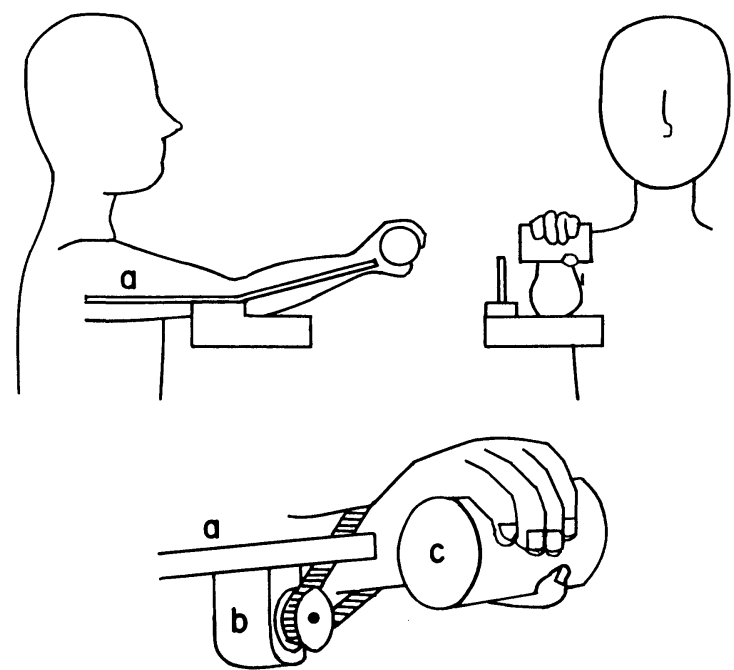

Fig. 1. Experimental situation of EMG recording during a to-and-fro motion from prone to supine of the forearm at 30 degrees flexion of the elbow. A cylindrical object (c) is held in the hand. A potentiometer (b) is fitted on a goniometer (a) to indicate which motion is acted, supination or pronation. 
performed, supination or pronation (Fig. 1). Mean amplitudes of averaged EMGs during keeping the forearm supine (MA) were measured using a digitizer (KD4300; GRAPHTEC, Tokyo).

\section{RESULTS}

\section{ENS study}

Movements of elbow flexion and forearm supination were induced simultaneously by ENS to each of BiL or BiS (Figs. 2,3) in six out of seven volunteers (Table 1). In the remainder, ENS to BiS induced elbow flexion and forearm supination as well as in the other volunteers but ENS to BiL induced elbow flexion only (Fig. 4). Slight medial rotation of the shoulder joint were induced by ENS to BiS (Fig. 3-b) in two volunteers and ENS to BiL in one volunteer.

\section{EMG study}

Appearance or disappearace, and quantities of EMG activities during PSmovement with the load in individual volunteers depended on elbow angles (Fig. 5 ). When the movement was performed with keeping the elbow at the maximum extension, and 30 and 60 degrees of flexion, both BiL and BiS showed EMG activities in all volunteers. When the movements were performed with keeping the elbow at 90 and 120 degrees of flexion and the maximum flexion, BiL or BiS in three out of eight volunteers showed no EMG activities throughout PSmovement. When EMG activities of BiL or BiS appeared during the movement, they usually increased and decreased accompanied with a degree of forearm

TABLE 1. ENS induced movement of the elbow joint

\begin{tabular}{ccllc}
\hline Name & Sex & Muscle & Flexion & Supination \\
\hline M.Y. & F & BiL & + & + \\
& & BiS & + & + \\
K.Y. & F & BiL & + & + \\
& & BiS & + & + \\
M.H. & F & BiL & + & + \\
& & BiS & + & + \\
M.T. & F & BiL & + & + \\
& & BiS & + & + \\
J.S. & F & Bil & + & - \\
& & BiS & + & + \\
H.F. & M & BiL & + & + \\
& & BiS $^{\mathrm{a}}$ & + & + \\
Y.H. & M & BiL $^{\mathrm{a}}$ & + & + \\
& & BiS $^{\mathrm{a}}$ & + & + \\
\hline
\end{tabular}

a Medial rotation of the shoulder joint was induced. 

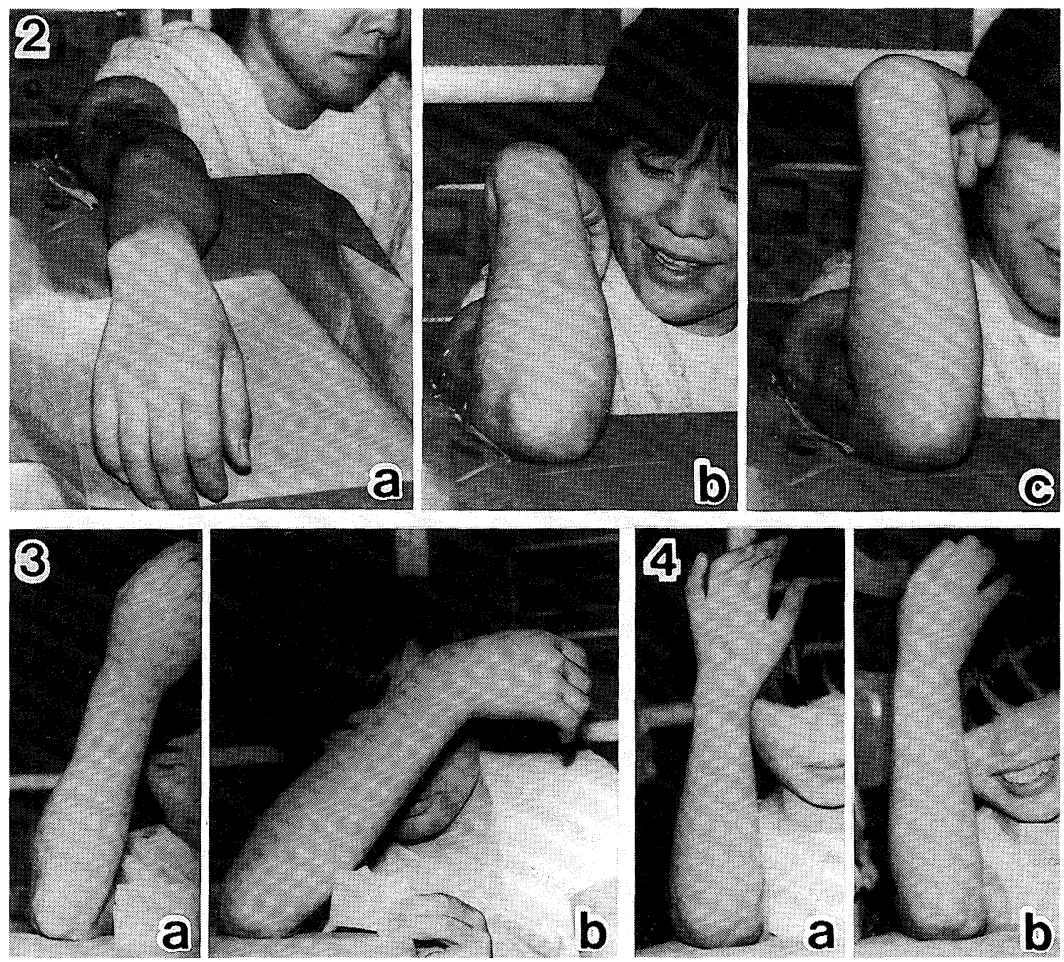

Fig. 2. Experimental posture and ENS-induced movements of the upper extremity in a volunteer (M.Y., female). Before ENS, the volunteer relaxes the upper extremity on a table with the shoulder flexed to about 90 degrees, and the elbow extended and the forearm pronated (a). Each ENS to BiL (b) and BiS (c) induced both movements of elbow flexion and forearm supination.

Fig. 3. ENS-induced movements of the upper extremity in a volunteer (H.F, male). ENS to BiL induced movements of elbow flexion and forearm supination (a). Medial rotation of the shoulder was induced by ENS to BiS in addition to elbow flexion and forearm supination (b).

Fig. 4. ENS-induced movements of the upper extremity in a volunteer (J.S., female). ENS to BiL (a) resulted in only an induction of elbow flexion, while ENS to BiS induced elbow flexion and forearm supination.

supination. During keeping the forearm in the prone before and after the movement, BiL and BiS showed slight or no EMG activities. Gradual increases of EMG activities occurred after supination were started and continued until the end of supination. When the forearm was keeping in the supine, EMG activities were seen to be a plateau. Gradual decrease of EMG activities were observed from beginning to end of pronation. These fluctuations provided bell-shaped or trapezoidal waveforms of averaged EMGs, while each amplitude of the averaged EMGs, that is to say the highest of the bell or trapezoid, varied with changing elbow angles. 


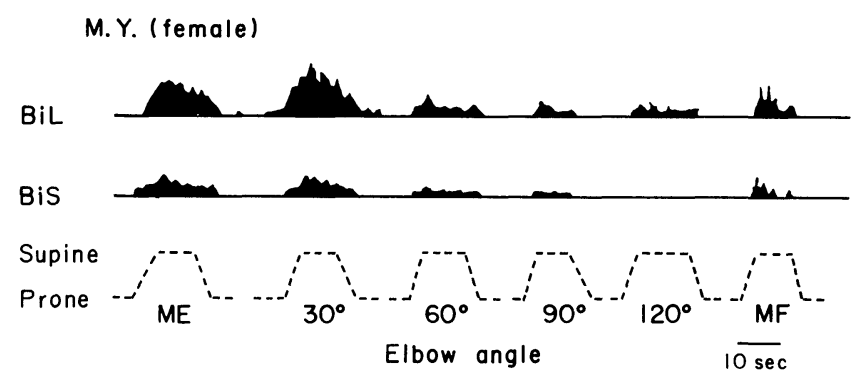

K. Y. (female)
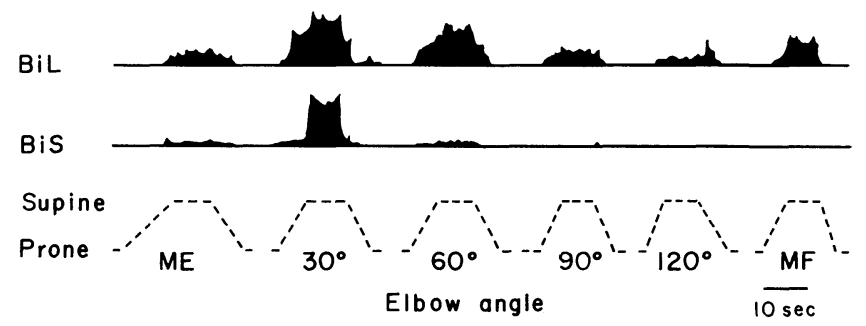

Fig. 5. Averaged EMGs of BiL and BiS during experimental movements in two volunteers (M.Y., K.Y., female). Broken lines indicate directions of the movements. ME, maximum extension; MF, maximum flexion.

In order to compare patterns of changes in quantities of EMG activities to changing elbow angles during PS-movement between BiL and BiS in each volunteer, and among the volunteers, mean amplitudes of averaged EMGs during keeping the forearm supine (MA) were measured. For standardization, MAs were indicated by a ratio to the maximum $\mathrm{MA}$ of $\mathrm{BiL}$ and $\mathrm{BiS}$ in each volunteer (Fig. 6). In seven out of eight cases, the maximum MA of BiL and BiS was observed during the movement with keeping the elbow joint in 30 degrees of flexion or the maximum extension. Three volunteers showed large MAs of BiL or BiS during the movement with the maximum elbow flexion (M.T., J.I. and K.M. in Fig. 6). In contrast, three of the others showed slight or no MAs of BiL or BiS at the maximum flexion (K.Y., M.H. and K.U. in Fig. 6). Comparisons of MAs found that patterns of changes in quantities of EMG activities to changing elbow angles varied from BiL to BiS in each volunteer, and from individual to individual.

\section{Discussion}

The present ENS study demonstrated that contractions of the biceps brachii including BiL and BiS induced movements of elbow flexion and forearm supination in all volunteer. This result agrees with actions of this muscle on the elbow joint described in most textbooks of anatomy (Sinclair 1981; Basmajian 1982; Clemente 1985; Williams et al. 1989). In the biceps brachii, muscle berries of BiL and BiS fuse at about halfway down the upper arm and the muscle is inserted by a cord-like tendon into the radial tuberosity (RT), and by a sheet-like tendon 

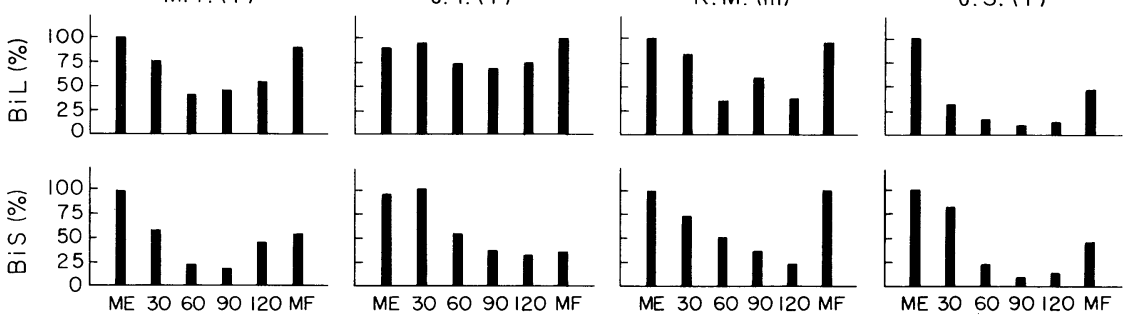

Elbow angle
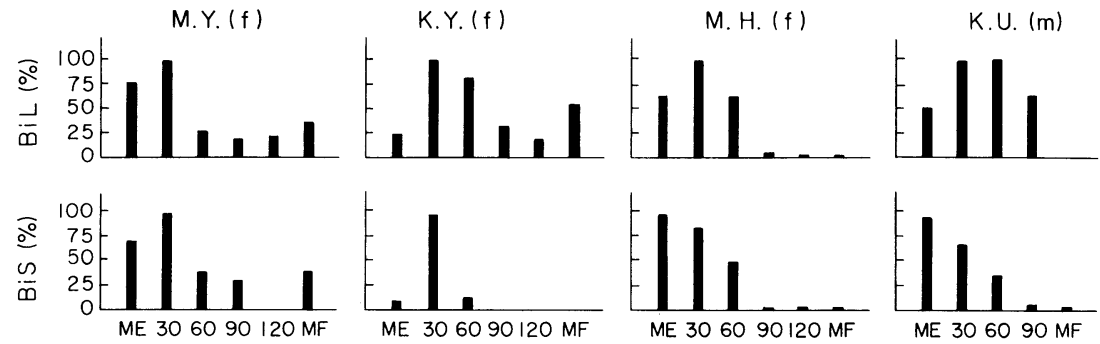

Elbow angle

Fig. 6. Changes in mean amplitudes of averaged EMGs during keeping the forearm supine (MA) to changing elbow angles in all volunteer. MA was indicated by a percentage to the maximum $\mathrm{MA}$ of $\mathrm{BiL}$ and $\mathrm{BiS}$ in each volunteer. Note that BiL and BiS in each volunteer showed different patterns of changes in MA and that the volunteers had nothing common in the pattern.

(Aponeurosis m. bicipitis brachii; Ap) into the antebrachial fascia (Basmajian 1982). From experimental tractions of RT and Ap in fresh cadavers, we found that RT traction induced elbow flexion and forearm supination but Ap traction resulted in induction of elbow flexion only (Naito et al. 1989). Furthermore, anatomical dissections studies indicated an existence of wide individual differences between insertions of BiL and BiS muscle fibers (Landau and Scheuchzer 1923; Naito et al. 1989). The reason why ENS to BiL did not induce supination in one volunteer is that most BiL fibers of this volunteer might insert into the antebrachial fascia as Ap.

It is well known that the biceps brachii dose not act on the prone forearm (Beevor 1903; Sullivan et al. 1950; Basmajian and Latif 1957; Pauly et al. 1967; Basmajian and Deluca 1985; Naito et al. 1991). From EMG study, Basmajian and Latif (1957) reported that the biceps brachii was generally active in flexion while keeping the forearm supine without a load and semiprone with a load (of 2 pound) but this muscle played little if any role in flexion, in maintenance of elbow flexion, and in antagonistic action (extension) while keeping the forearm prone with the load. In the present EMG study, BiL and BiS showed slight or no EMG activities during keeping the forearm prone with a load (of $1.2 \mathrm{~kg}$ ). This result agrees with that of Basmajian and Latif in regard to maintenance of flexion. The 
present EMG study also demonstrated that BiL and BiS showed an increase and a decrease of EMG activities accompanied by a degree of forearm supination. This observation clearly indicated an above-mentioned character of the biceps brachii as well as Basmajian and Latif's report. (Basmajian and Latif 1957).

EMG studies of the elbow flexors and extensors indicated an existence of wide individual differences in activities of these muscles during elbow movements (Sullivan et al. 1950; Basmajian and Latif 1957; Naito et al. 1991). In the present EMG study, averaged EMGs of BiL and BiS in the volunteers usually showed bell-shaped or trapezoidal waveforms during PS-movement. However, the pattern of changes in quantities of EMG activities to changing elbow angles in $\mathrm{BiL}$ and $\mathrm{BiS}$ different from individual to individual. Considering the results of the present ENS study, it is thought that each human subject has an individual use of the biceps brachii for forearm supination and pronation movements while the actions of the muscle on the elbow joint are similar among the subjects. Further detailed analysis of the movement, i.e., simultaneous ENS to the biceps brachii and the other muscles or simultaneous EMG recording of the elbow flexors and extensors, and the forearm pronators and supinators, are needed to use FES to the biceps brachii for controlling forearm supination movements.

\section{Acknowledǵments}

This work was partly supported by Grant-in Aid for Scientific Research from the Ministry of Education, Science and Culture of Japan (No. 61890007, 62460130, 63850081, 01870001). We wish to thank Mr. Michio Miyata and Miss Kumiko Yokouchi for their excellent assistance. Thanks are also tendered to members of our FES research group and students of Shinshu University School of Medicine for their cooperation.

\section{References}

1) Basmajian, J.V. (1982) Primary Anatomy. 8th ed., Williams and Wilkins, Baltimore, pp. 149-155.

2) Basmajian, J.V. \& Latif, A. (1957) Integrated actions and functions of the chief flexors of the elbow. J. Bone Joint Surg., 39-A, 1106-1118.

3) Basmajian, J.V. \& Deluca, C.J. (1985) Muscle Alive: Their Functions Revealed by Electromyography. 5th ed., William and Wilkins, Baltimore, pp. 265-289.

4) Beevor, C.E. (1903) Croonian lectures on muscular movements and their representation in the central nervous system. Lancet, 1, 1715-1724.

5) Clemente, C.D. (1985) Gray's Anatomy of the Human Body. 30th American ed., Lea and Febiger, Philadelphia, pp. 527-528.

6) Fukamachi, H., Handa, Y., Naito, A., Ichie, M., Yajima, M., Ushikoshi, K., Tsuchiya, M., Matsushita, N. \& Hoshimiya, N. (1987) Improvement of finger movement by intrinsic muscles stimulation of the hand. Proc. Ann. Conf. IEEE/EMBS, 9, 361362.

7) Handa, Y. \& Hoshimiya, N. (1987) Functional electrical stimulation for the control of the upper extremities. Med. Prog. Technol., 12, 51-63.

8) Handa, Y., Naito, A., Ichie, M., Handa, T., Matsushita, N. \& Hoshimiya, N. (1987) EMG-based stimulation patterns of FES for the paralyzed upper extremities. In: Proc. 9th Int. Symp. on External Control of Human Extremities edited by D. Popovic, 
Yugoslav. Committee for Electronics and Automation, Bergrade, pp. 329-337.

9) Handa, Y., Hoshimiya, N., Iguchi, Y. \& Oda, T. (1989) Developments of percutaneous intramuscular electrode for multi-channel FES system. IEEE Trans. Biomed. Eng., 36, 705-710.

10) Hoshimiya, N., Naito, A., Yajima, M. \& Handa, Y. (1989) A respiration controlled FES system for muti-joint upper extremity. IEEE Trans. Biomed. Eng., 36, 754-760.

11) Ichie, M., Handa, Y., Naito, A., Handa, T., Matsushita, N. \& Hoshimiya, N. (1986) EMG analysis of the thumb and its application to ENS. Proc. Ann. Conf. IEEE/ EMBS, 8, 538-540.

12) Kapandji, I.A. (1986) Physiologie Articulaire I: Membre Superieur. 1st ed., Ishiyaku Publ. Co., Tokyo, pp. 132-165. (in Japanese)

13) Landau, E. \& Scheuchzer, W. (1923) Beitrag zur Kenntnis des Lacertus fibrosus des M. biceps brachii. Mitteilungen der Naturf. Gesellschaft in Bern, 33-38.

14) Liberson, W.T., Holmquest, H.J. \& Scot, D. (1961) Functional electrotherapy: Stimulation of peroneal nerve synchronized with the swing phase of the gait of hemiplegic patients. Arch. Phys. Med., 42, 101-105.

15) Marsolais, E.B. \& Kobetic, R. (1983) Functional walking in paralyzed patients by means of electrical stimulation. Clin. Orthop., 175, 30-36.

16) Matsushita, N., Handa, Y., Ichie, M., Naito, A., Handa, T. \& Hoshimiya, N. (1986) Analysis of wrist movements and its application to FNS. Proc. Ann. Conf. IEEE/ $E M B S, 8,618-619$.

17) Naito, A., Handa, Y., Ichie, M., Handa, T., Matsushita, N., Yazima, M., Fukamachi, H., Tsuchiya, M. \& Hoshimiya, N. (1987) EMG analysis of elbow movements and its application to FNS. Proc. Ann. Conf. IEEE/EMBS, 9, 46-47.

18) Naito, A., Handa, Y., Yajima, M., Ishikawa, S., Okubo, K. \& Hoshimiya, N. (1988) Development of a portable FES system for the paralyzed extremities. Proc. Ann. Conf. IEEE/EMBS, 10, 1669-1670.

19) Naito, A., Ichie, M., Shimizu, Y. \& Handa, Y. (1989) Analysis of the action of the biceps brachii. Acta Anat. Nippon (Abst.), 64, 373. (in Japanese)

20) Naito, A., Yajima, M., Fukamachi, H., Ushikoshi, K., Ichie, M., Handa, Y. \& Hoshimiya, N. (1990) Creation of neuromuscular stimulation data for functional electrical stimulation: Control of paralyzed elbow movements. Jpn. J. Med. Elect. Biol. Eng., 28, 50-55. (in Japanese with English abstract)

21) Naito, A., Shimizu, Y., Handa, Y., Ichie, M. \& Hoshimiya, N. (1991) Functional anatomical studies of the elbow movements. I. Electromyographic (EMG) analysis. Okajimas Folia Anat. Jpn., 68, 283-288.

22) Nathan, R.H. (1984) The development of a computerized upper limb electrical stimulation system. Orthopäde, 7, 1170-1180.

23) Pauly, J.E., Rushing, J.L. \& Scheving, L.E. (1967) An electromyographic study of some muscles crossing the elbow joint. Anat. Rec., 159, 47-54.

24) Sinclair, D.C. (1981) Muscle and Fascia. In: Cunningham's Textbook of Anatomy. 12th ed., Oxford University Press, London, pp. 322-323.

25) Sullivan, W.E., Mortensen, O.A., Miles, M. \& Green, L.S. (1950) Electromyographic studies of $\mathrm{m}$. biceps brachii during normal voluntary movement at the elbow. Anat. Rec., 107, 243-252.

26) Williams, P.L., Dyson, M., Bannister, L.H. \& Warwick, R. (1989) Gray's Anatomy. 37th ed., Churchill Livingstone, Edinburgh, pp. 614-615. 